\title{
Coulomb correlations of charged excitons in semiconductor quantum dots
}

\author{
D. Y. Oberli, M. Byszewski, B. Chalupar, E. Pelucchi, * A. Rudra, and E. Kapon \\ Laboratory of Physics of Nanostructures, Ecole Polytechnique Fédérale de Lausanne (EPFL), CH-1015 Lausanne, Switzerland
}

(Received 24 November 2008; revised manuscript received 2 September 2009; published 7 October 2009)

\begin{abstract}
The emission pattern of charged excitons in a semiconductor quantum dot (QD) is composed of a quadruplet of linearly polarized lines when a magnetic field is applied in a Voigt configuration. The orientation of the linear polarization of exciton emission is controlled by the orientation of the magnetic field in QDs with $\mathrm{C}_{3 \mathrm{v}}$ symmetry while for QDs with $\mathrm{C}_{2 \mathrm{v}}$ symmetry it is not. We demonstrate that the $g$ factor of holes is very sensitive to the dot shape asymmetry but that of electrons is not. By comparing the effective $g$ factors obtained for the neutral and charged excitons in the same quantum dot, we uncover the role of Coulomb correlations in these excitonic states. We show that the $C_{3 v}$ symmetry of pyramidal QDs makes them ideal candidates for implementing all-optical many-qubits gates based on electron spin as a quantum bit.
\end{abstract}

DOI: 10.1103/PhysRevB.80.165312

PACS number(s): 78.67.Hc, 71.70.Ej, 71.70.Gm, 78.55.Cr

Spin degree of freedom of carriers in semiconductor quantum dots (QDs) could serve as quantum bits to store ${ }^{1}$ and process ${ }^{2,3}$ information in spin-based devices. Recent experiments demonstrated essential steps toward the implementation of these concepts. Effective initialization of a spin state was achieved for either an electron ${ }^{4,5}$ or a hole $e^{6,7}$ confined to a single quantum dot. Rapid rotation of an electron spin was accomplished by driving coherently the two spin states with short optical pulses by means of stimulated spinflip Raman scattering. ${ }^{8}$ The coherent manipulation of a carrier $\operatorname{spin}^{9}$ in a spin-flip Raman process involves the creation of two energetically different spin states by applying a magnetic field. A key property of an electron (or a hole) confined to a QD is the effective $g$ factor that measures the Zeeman splitting of the ground state in an applied magnetic field and depends on the orientation of the field with respect to the symmetry axis of the QD. Even though the coherent manipulation of a single quantum dot spin was successively demonstrated, the extension of this scheme to quantum dot arrays seems to be a challenge as QDs must have a narrow distribution of emission energies and obey specific optical selection rules. It is thus necessary to investigate the homogeneity of the Zeeman splittings and the uniformity of the optical selection rules in QD arrays.

Zeeman splittings in QDs have been investigated by capacitance spectroscopy, ${ }^{10}$ by magnetophotoluminescence $\mathrm{e}^{11,12}$ and by transient nonlinear optical techniques. ${ }^{13}$ While transport techniques probe the $g$ factors of electronic states, optical techniques probe the Zeeman splittings of excitonic states. The optical anisotropy of QDs was also studied by applying a magnetic field in the Voigt geometry, which revealed a complex behavior due to shape asymmetry and strain present in self-assembled quantum dots (SAQDs). ${ }^{14-17}$

In principle, probing different charged excitonic states in QDs could yield different values of the Zeeman splittings if the Coulomb correlations between carriers altered the singleparticle wave function of the strongly confined carriers. Coulomb interactions between carriers confined in QDs are exemplified by the rich optical spectra of multiply charged excitonic complexes demonstrating the role of exchange interactions between electrons or holes. ${ }^{18,19}$ Despite the remarkable predictiveness of many-body calculations of these excitonic complexes remaining uncertainty about the real dot morphology prevents a positive identification of correlation effects in the experimental spectra of singly charged excitons. ${ }^{20-23}$

In this study, we present a systematic investigation of the emission spectra of excitonic complexes confined in QDs when an external magnetic field is applied in a direction perpendicular to the optical wave vector (Voigt configuration). The effective $g$ factors of an electron and a hole in individual QDs are obtained on the basis of a consistent analysis of the Zeeman splittings in many QDs. Our results reveal a large uniformity of the electron $g$ factors but a broad variation in hole $g$ factors when analyzing QDs emitting at nearly the same energy. By comparing the polarized emission spectra from the neutral and charged excitons in the same QD, we evidence a significant modification of the carrier $g$ factors that we attribute to Coulomb correlations within the few-particle excitonic complexes.

We use pyramidal $\mathrm{In}_{0.1} \mathrm{Ga}_{0.9} \mathrm{As} / \mathrm{Al}_{0.3} \mathrm{Ga}_{0.7} \mathrm{As}$ QDs that allow us to observe simultaneously the positively and the negatively charged excitons in the same photoluminescence (PL) spectrum. ${ }^{24}$ The measurements were performed on individual QDs at a temperature of $10 \mathrm{~K}$ using a microphotoluminescence setup. The cryostat containing the sample was inserted in the room-temperature bore of a superconducting magnet, which generates magnetic fields up to $6.5 \mathrm{~T}$. The PL was excited with a continuous-wave Ti:sapphire laser operating at $700 \mathrm{~nm}$ and dispersed in a spectrograph equipped with a Si charged-coupled device detector. The spectral resolution was $40 \mu \mathrm{eV}$ and the spectral precision was $\pm 5 \mu \mathrm{eV}$ by fitting the spectral line shape. We found that the linewidth of an excitonic transition lay between 80 and $110 \mu \mathrm{eV}$ for the investigated QDs.

A typical PL spectrum of a pyramidal QD at zero magnetic field is presented in Fig. 1(a). It consists of a series of four major lines, which correspond to the recombination of the negatively charged exciton $\left(\mathrm{X}^{-}\right)$of a biexciton $(2 \mathrm{X})$, of a neutral exciton $(\mathrm{X})$, and of the positively charged exciton $\left(\mathrm{X}^{+}\right)$ordered by increasing energies. ${ }^{24}$ The linearly polarized emission spectra evidence the existence of a doublet for both the neutral exciton and the biexciton while the charged exciton lines do not feature any resolvable splittings. Consequently, we attribute the splitting of $\mathrm{X}(2 \mathrm{X})$ to the anisotropic part of the electron-hole $(e-h)$ exchange interaction, which 


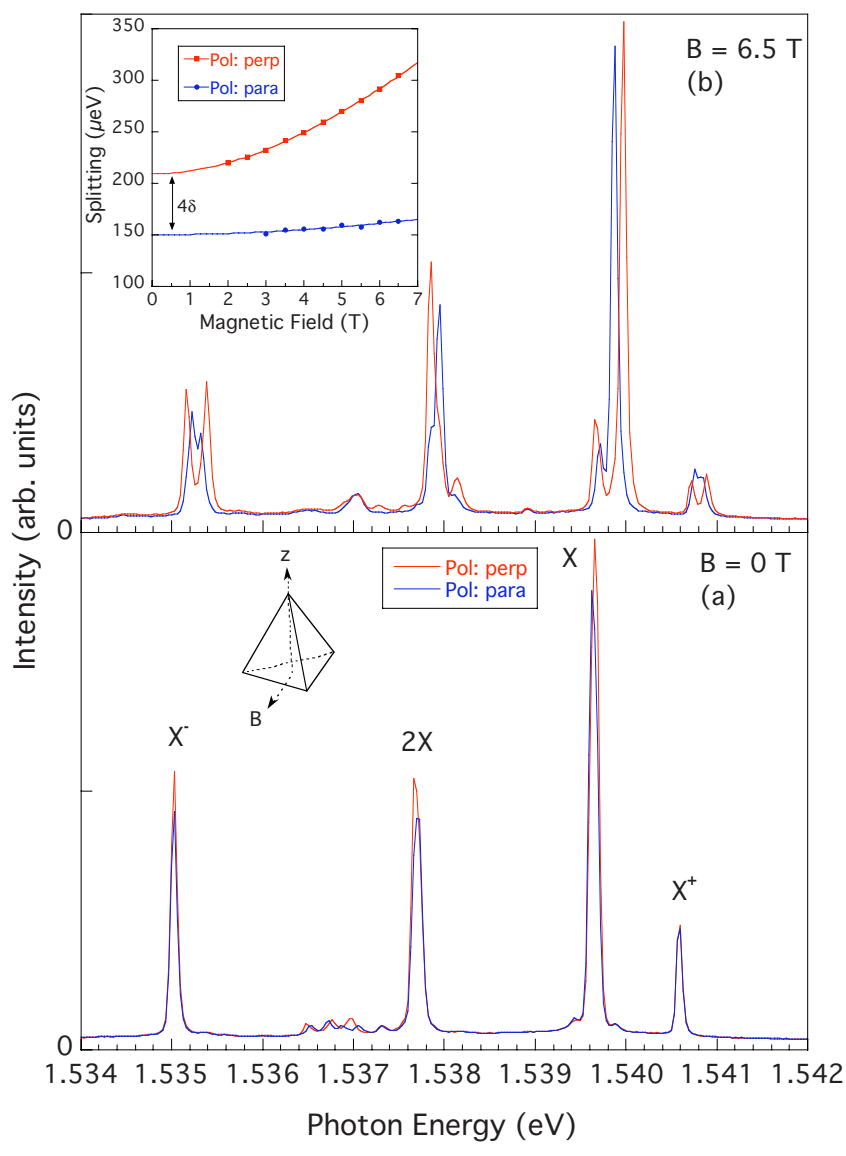

FIG. 1. (Color online) Polarized PL spectra of an isolated quantum dot measured (a) without and (b) with a magnetic field of $6.5 \mathrm{~T}$ in the Voigt configuration. The linear polarization is oriented in a direction parallel or perpendicular to the magnetic field. Upper inset: Zeeman energy splitting between the bright and dark excitons for each of the linear polarizations. Lines are fits using expressions given in the text. Lower inset: sketch of pyramidal dot showing the photon emission direction $(\mathbf{k} \|[111])$ and the orientation of the magnetic field $(\mathbf{B} \|[1-10])$.

has been thoroughly studied in SAQDs. ${ }^{11,13}$ The weaker lines in the PL spectra have been previously identified as emission from excitonic complexes wherein one of the holes occupies an excited state of the QD. ${ }^{25}$

The application of a magnetic field in the Voigt configuration leads to striking modifications of the PL spectra of the QD as shown in Fig. 1(b), where we display the linear polarization spectra of the QD under an applied magnetic field of $B=6.5 \mathrm{~T}$. The emission is linearly polarized in a direction that is either parallel or perpendicular to the field direction. For a given orientation of the polarization, the emission of the charged excitons consists of a doublet of lines of nearly equal intensities whereas that of the neutral exciton (biexciton) consists of a doublet with a weak line on the low- (respectively, high-) energy side of a dominant line. This latter emission pattern is similar to the well-known signature (Ref. 11) of the hybridization between the "dark" exciton states of total angular momentum $\left(m_{z}= \pm 2\right)$ and of the "bright" exciton states of angular momentum $\left(m_{z}= \pm 1\right)$ that is caused by a magnetic field when applied in a direction perpendicular to the $z$ axis of a QD [see inset of Fig. 1(a)]. In the inset of Fig.

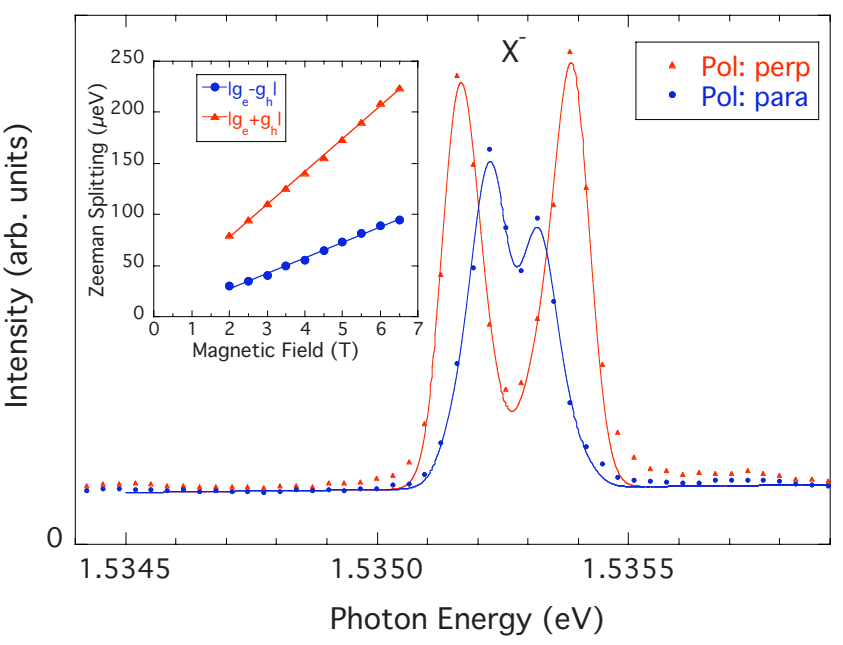

FIG. 2. (Color online) Emission spectra of $\mathrm{X}^{-}$at a magnetic field of $6.5 \mathrm{~T}$ displaying a quadruplet of linearly polarized transitions. Lines correspond to fits of the data with Gaussians of identical width. Inset: Zeeman splittings plotted versus magnetic field.

1(b), we display the measured energy splitting of the neutral exciton doublet versus the magnetic field for each polarization of the emission. It increases nearly quadratically with the magnetic field. The magnetic field dependence of these splittings is determined by the Zeeman interaction between the spin of the carriers and $B$ and by the $e-h$ exchange interaction as explained later.

We will first focus on the emission patterns of the charged excitons. In striking contrast to the neutral exciton behavior in the magnetic field, we observe that the doublet components have nearly equal intensities at all values of the magnetic field: at $B=6.5 \mathrm{~T}$, e.g., the spectra of the negatively charged exciton are expanded in Fig. 2. Moreover, we find that the splitting of each charged exciton doublet increases linearly with $B$ as shown in the inset for the case of $\mathrm{X}^{-}$. These splittings were determined very precisely (error equal to $\pm 5 \mu \mathrm{eV}$ ) on the basis of a fit of the doublets to Gaussian line shapes. The Gaussian broadening is caused by the spectral diffusion of the line due to charge fluctuation in the QD vicinity.

The behavior of the charged exciton states in a magnetic field, their quadruplet emission pattern, and their polarization properties, are fully determined by the Zeeman interaction alone. In a singly charged excitonic complex, the $e-h$ exchange interaction does not contribute to the splitting irrespectively of the QD symmetry because of the Kramers degeneracy of the charged exciton states. ${ }^{26}$ To explain the splitting pattern of the charged exciton emission, we exploit the $\mathrm{C}_{3 \mathrm{v}}$ point group symmetry of the pyramidal QD structure and detect photons emitted in the direction of its rotation axis $(z \|[111])$. The Zeeman Hamiltonian of a carrier is given by the general expression using the method of invariants: ${ }^{27,28}$ $H_{Z}^{c}= \pm \frac{1}{2} \sum_{i=x, y, z} g_{i}^{c} \mu_{B} \sigma_{i} B_{i}$, where $c$ stands for the carrier type, $\mu_{B}$ is the Bohr magneton, $\sigma_{i}$ are the Pauli spin matrices, and $g_{i}^{c}$ is a diagonal tensor representing the effective $g$ factors of a carrier (minus sign applies to a hole to be consistent with the sign of the Zeeman Hamiltonian in the hole representation $\left.{ }^{29}\right)$. It is important to note that $g_{x}^{c}=g_{y}^{c}$ since $B_{x}$ 
and $B_{y}$ transforms under $\mathrm{C}_{3 \mathrm{v}}$ according to the same representation $\left(\Gamma_{3}\right)$ and that $g_{z}^{c} \neq g_{x}^{c}$ since $B_{z}$ transforms according to another representation $\left(\Gamma_{2}\right)$. Here, $x$ and $y$ are parallel to the symmetry axes given, respectively, by [1-10] and [11-2]. The expression of the Zeeman interaction applies equally to electron and hole eigenstates when these belong to the twodimensional representation of $\mathrm{C}_{3 \mathrm{v}}\left(\Gamma_{4}\right) \cdot{ }^{30,31}$ For a magnetic field oriented perpendicularly to one of the $\sigma_{\mathrm{v}}$ symmetry planes (e.g., $B \|[1-10])$ the Zeeman Hamiltonian is nondiagonal in the spinor basis $\left\{\left|\frac{1}{2},+\frac{1}{2}\right\rangle,\left|\frac{1}{2},-\frac{1}{2}\right\rangle\right\}$ corresponding to eigenstates of the angular momentum operator $J_{z}$, where $z$ indicates the quantization axis taken along [111]. In diagonalizing the Zeeman Hamiltonian the spinor eigenvectors for each carrier type assume the form $\frac{1}{\sqrt{2}}\left[\left|\frac{1}{2}, \frac{1}{2}\right\rangle \pm\left|\frac{1}{2},-\frac{1}{2}\right\rangle\right]$ and the Zeeman energies are given by $\pm \frac{1}{2} g_{x}^{c} \mu_{B} B_{x}$. From these eigenstates, we can simply derive the polarization selection rules in the dipole approximation for a photon emitted along $z$ using the Wigner-Eckart theorem for simply reducible point groups: four linearly polarized optical transitions with equal strength are predicted, two of them being polarized perpendicularly to the magnetic field direction and split by $\Delta E_{\perp}=\mu_{B}\left|g_{x}^{e}+g_{x}^{h}\right| B_{x}$ and the other two being polarized parallel to $B$ and split by $\Delta E_{\|}=\mu_{B}\left|g_{x}^{e}-g_{x}^{h}\right| B_{x}$. The polarization rules and the linear dependence of the splitting with $B$ are perfectly confirmed by our experimental data on this QD, as shown in Fig. 2 for the case of $\mathrm{X}^{-}$, and also on ten other QDs. The symmetry of the dot structures was confirmed by rotating the sample by $60^{\circ}$ around the $z$ axis of the pyramid. The same splitting pattern was observed as described above since this rotation is equivalent to reversing the direction of the magnetic field.

From the measurement of the Zeeman splittings at $B=6.5 \mathrm{~T}$ we directly determine the modulus of the effective $g$ factor for each carrier type. The data in Fig. 2 yield $\left|g_{x}^{e}\right|=(0.15 \pm 0.01)$ and $\left|g_{x}^{h}\right|=(0.42 \pm 0.01)$ in the case of $\mathrm{X}^{-}$. The attribution of the value 0.15 to the electron $g$ factor is fully consistent with the determination made in a set of ten other QDs (see Fig. 4). An important point is that the relative signs of the $g$ factors can always be specified in a Voigt configuration. For the particular QD of Fig. 1, the signs are identical since the largest Zeeman splitting is obtained for a linear polarization that is perpendicular to $B$.

Since we observe both the emission of $\mathrm{X}^{+}$and $\mathrm{X}^{-}$in the same dot we can test whether the presence of an additional carrier in the dot modifies the value of the effective $g$ factors. We find indeed that the Zeeman splitting of $\mathrm{X}^{+}$is smaller than that of $\mathrm{X}^{-}$in each of the two polarizations. This means that the effective $g$ factors depend on the sign of the charged exciton. Following the analysis done for $\mathrm{X}^{-}$, we obtain from the quadruplet splittings of $\mathrm{X}^{+}$(see Fig. 3), $\left|g_{x}^{e}\right|=(0.14 \pm 0.01)$ and $\left|g_{x}^{h}\right|=(0.38 \pm 0.01)$. Although the differences between the two sets of carrier $g$ factors are small, the measured Zeeman splittings for $\mathrm{X}^{+}$differ significantly from those of $\mathrm{X}^{-}$as shown in Fig. 1(b). These observations were confirmed in the magneto-optical spectra of ten other pyramidal QDs selected from the same sample for their emission within a narrow energy range (5 meV). In Fig. 4, we compare the carrier $g$ factors measured in these QDs for both $\mathrm{X}^{+}$and $\mathrm{X}^{-}$. For the electron $g$ factor, we observe a

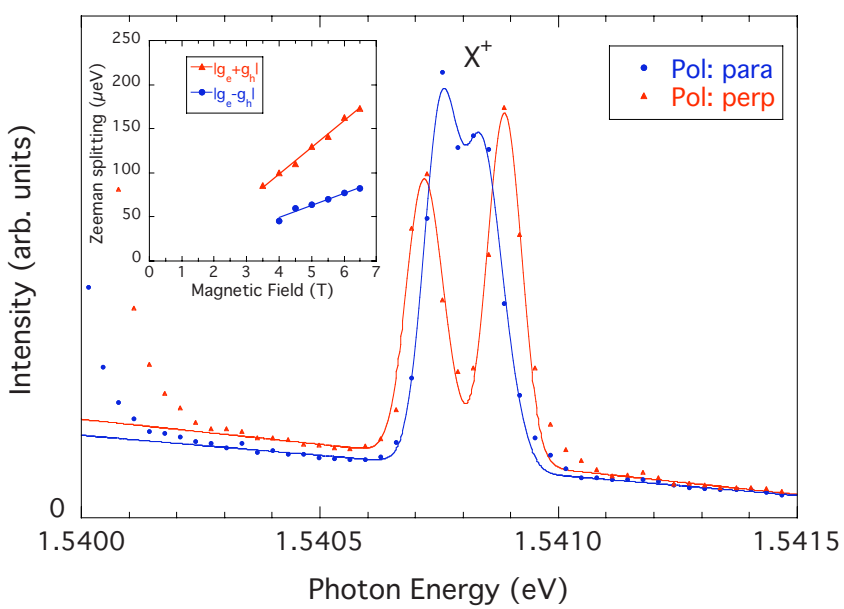

FIG. 3. (Color online) Emission spectra of $\mathrm{X}^{+}$at a magnetic field of $6.5 \mathrm{~T}$ displaying a quadruplet of linearly polarized transitions. Lines correspond to fits of the data with Gaussians of identical width. Inset: Zeeman splittings plotted versus magnetic field.

marked difference between the values obtained from the splittings of $\mathrm{X}^{+}$and $\mathrm{X}^{-}$. The modulus of the electron $g$ factor of $\mathrm{X}^{-}$is always larger than that of $\mathrm{X}^{+}$: the mean value being $\left\langle\left|g_{x}^{e}\right|\right\rangle=0.18$ for $\mathrm{X}^{-}$and $\left\langle\left|g_{x}^{e}\right|\right\rangle=0.12$ for $\mathrm{X}^{+}$; the standard deviation being $\sigma=0.02$. This suggests that the presence of an additional carrier alters the single-particle electron wave function and, thus, modifies the electron $g$ factor measured from either $\mathrm{X}^{+}$or $\mathrm{X}^{-}$. In the case of holes, the effective $g$ factor measured from $\mathrm{X}^{-}$can be either smaller or larger than that of $\mathrm{X}^{+}$(Fig. 4). Similarly, this evidences a distortion of the hole wave function in each QD. Additionally, we find a broad distribution of effective $g$ factors for holes, which contrasts with the narrow distribution found for electrons. Given that the error on the $g$ factor is small, we infer from the distribution width that the hole $g$ factor depends sensitively on the QD by a change of its shape or its volume. A change in $\mathrm{QD}$ volume would result in a correlation between the emission energy of the exciton and the hole $g$ factor. However, no such correlation was found. On the other hand, a

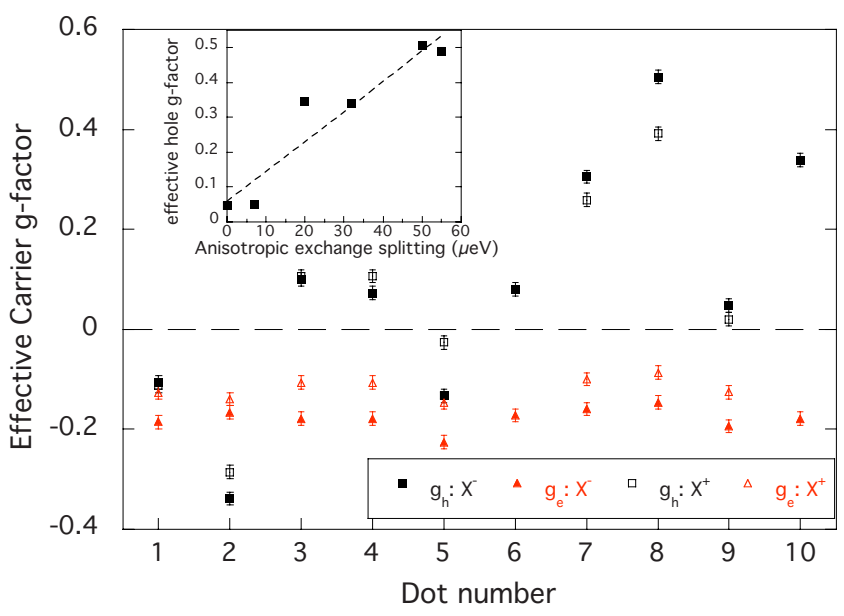

FIG. 4. (Color online) Effective carrier $g$ factors for the charged excitons measured for a set of ten dots. Inset: hole effective $g$ factor from $\mathrm{X}^{-}$plotted versus anisotropic exchange term $2\left|\delta-\delta^{*}\right|$. 
breaking of the axial symmetry of the QD will alter its shape and give rise to an anisotropic $e-h$ exchange interaction. This interaction is known ${ }^{32,33}$ to be a sensitive probe of a QD asymmetry. It is evidenced by a splitting of the neutral exciton emission into a doublet with linearly polarized components. Experimentally, we find indeed a strong correlation between the magnitude of this splitting at zero magnetic field and the hole $g$ factor, as shown in inset of Fig. 4. The larger the hole $g$ factor, the larger the anisotropic $e-h$ exchange splitting is. This behavior confirms that the broad distribution of hole $g$ factors results from a shape modification of the QD. This deduction is corroborated by theoretical calculations, ${ }^{34}$ which predict a large increase in the in-plane hole $g$ factor resulting from a breaking of axial symmetry. Moreover, our observation of a narrow distribution of electron $g$ factors is in good agreement with these calculations and those performed by Sheng et al. ${ }^{35}$

Magneto-PL experiments performed in the Voigt configuration yield the modulus of the carrier $g$ factors but not the sign. The attribution of a negative sign to the electron $g$ factor in the plane perpendicular to the growth axis (see Fig. 4) is thus a choice for sake of consistency. Indeed, by performing experiments in the Faraday configuration in similar pyramidal QDs, we could determine that $g_{z}^{e}$ is negative when $\mathbf{B}$ is oriented along the [111] growth axis. ${ }^{36} \mathrm{We}$ wish to emphasize, however, that the sign of the hole $g$ factor can be specified relatively to that of the electron $g$ factor in the Voigt configuration. In these pyramidal QDs, we find a surprising result: the in-plane electron and hole $g$ factors can have the same or an opposite sign. This effect might be explained by a deformation of the pyramid shape. If the Kramers degenerate hole ground states were to belong to the onedimensional representations $\left(\Gamma_{5}\right.$ and $\left.\Gamma_{6}\right)$ then the expected value of the hole $g$ factor would be zero due to symmetry considerations. A deformation of the pyramid might then yield a finite Zeeman splitting of the hole states in a magnetic field applied perpendicularly to [111]; the hole $g$ factor could then take either one or other sign depending on the type of symmetry breaking. An assignment of the symmetry of the hole ground states to $\Gamma_{5}$ and $\Gamma_{6}$ is thus entirely consistent with the variation in the hole $g$ factor with the anisotropic exchange splitting observed in Fig. 4, which takes a nearly zero value at the origin. Although the sign of the in-plane electron $g$ factor is undetermined experimentally, the attribution of a negative sign cannot be justified on the basis of existing theoretical works (Refs. 34 and 35) since an inconsistent prediction of the sign of the electron $g$ factor is made in strained InAs/GaAs QDs. The sign of the electron $g$ factor was specified only recently as being negative in InGaAs/GaAs-SAQDs using the dynamic nuclear polarization technique. ${ }^{37}$

The influence of an extra carrier in a QD on the modulus of the carrier effective $g$ factors is a consequence of the Coulomb interaction between carriers. As shown in Fig. 1 the Zeeman splitting for a given orientation of the polarization depends on the sign of the charged exciton. We find that the Zeeman splittings of $\mathrm{X}^{-}$are always larger than those of $\mathrm{X}^{+}$ for all the investigated QDs. In contrast, we measured identical Zeeman splittings in the Faraday configuration for both $\mathrm{X}^{-}, \mathrm{X}^{+}$, and $\mathrm{X}$ when $\mathbf{B}$ was oriented parallel to the growth axis (Ref. 36). Similar observations have been previously reported in studies of InAs-SAQDs. ${ }^{38,39}$ We infer from the sensitivity of the Zeeman splittings to the sign of the charged exciton that there is a significant degree of Coulomb correlation between the three carriers composing these excitonic complexes.

In order to corroborate this interpretation, we analyze the Zeeman splittings of the neutral exciton measured in the Voigt configuration. In the inset of Fig. 1 we observe a quadratic dependence of the Zeeman splittings on the strength of $B$. This dependence with $B$ is well accounted for by adding to the Zeeman Hamiltonian the $e-h$ exchange interaction written in the form (Ref. 27): $H_{\text {exch }}=\frac{1}{2} \delta_{\mathrm{o}} \sigma_{z}^{e} \cdot \sigma_{z}^{h}+\delta^{*} \sigma_{x}^{e} \cdot \sigma_{x}^{h}+\delta \sigma_{y}^{e} \cdot \sigma_{y}^{h}$, where $\sigma_{i}$ are the Pauli spin matrices for an electron or a hole. ${ }^{40}$ The first term in this expression is the isotropic exchange interaction that splits the neutral exciton into two doubly degenerate states, which are often referred to as the doubly degenerate bright states and the doubly degenerate dark states when the symmetry is $\mathrm{D}_{2 \mathrm{~d}}$. The second and third terms are the anisotropic exchange interactions that lift the degeneracy of these states without admixing them. We remark that the coefficients describing the strength of the anisotropic exchange terms are strictly equal for QDs with $\mathrm{C}_{3 \mathrm{v}}$ symmetry ${ }^{41}$ but will be different if the symmetry is lower or if the pyramidal shape is distorted. We do not need to include additional $e-h$ exchange terms since a mixing of the bright and dark states is not evidenced in the PL in the absence of a transverse magnetic field. The energy splittings of the doublets are derived by direct diagonalization of the spin Hamiltonian of an $e-h$ pair, $H_{s}=H_{z}^{e}+H_{z}^{h}+H_{\text {exch. }}$ Their expressions are given by $\Delta E_{\|(\perp)}=\sqrt{\left(\delta_{\mathrm{o}} \mp 2 \delta\right)^{2}+\left(g_{e}^{x} \mp g_{h}^{x}\right)^{2} \mu_{B}^{2} B_{x}^{2}}$, corresponding to a linear polarization of the doublet being either parallel $(\|)$ or perpendicular $(\perp)$ to the magnetic field (upper sign in the equation corresponds to the parallel polarization). We determine the effective carrier $g$ factors for $X$ by fitting the measured energy splittings with these expressions. From these fits [see inset of Fig. 1(b)], we obtain the values of $\left|g_{x}^{e}\right|=(0.206 \pm 0.006), \quad\left|g_{x}^{h}\right|=(0.376 \pm 0.006), \quad \delta_{0}=180 \mu \mathrm{eV}$, and $\delta=15 \mu \mathrm{eV}$. Experimentally, the neutral exciton emits a doublet of lines of equal intensities, with a splitting at zero magnetic field given by $2\left(\delta-\delta^{*}\right)=55 \mu \mathrm{eV}$, which yields $\delta^{*}=-12.5 \mu \mathrm{eV}$. By recording the spectra of other dots emitting closely in energy $( \pm 1 \mathrm{meV})$, we find a small dispersion of the isotropic exchange energy around an average value of $(196 \pm 10 \mu \mathrm{eV})$ whereas the anisotropic exchange energy, $\delta$, varies over the range $[0-15 \mu \mathrm{eV}]$. The observed constancy of the isotropic part of the exchange energy is understood because it is proportional to the probability for the electron and the hole to be at the same site, ${ }^{42}$ this probability remaining constant if the emission energy is unchanged. As the anisotropic part of exchange interaction is a sensitive probe of the QD asymmetry, it is then expected that a distortion of the QD away from a perfect pyramidal shape will introduce a splitting of the bright exciton states [given by $2\left(\delta-\delta^{*}\right)$ ] as evidenced experimentally in the inset of Fig. 4. Our measurements are in qualitative agreement with the theoretical analysis of the excitonic fine structure developed for $\mathrm{In}_{1-x} \mathrm{Ga}_{x} \mathrm{As} / \mathrm{GaAs}$ SAQDs. ${ }^{43,44}$ Moreover, we notice that the 
experimental splitting energy between the dark exciton states, given by $2\left(\delta^{*}+\delta\right)=(5 \pm 4) \mu \mathrm{eV}$, takes a very small value as calculated theoretically for flat QDs with $C_{2 v}$ symmetry (see Ref. 44). However, the exciton fine structure arising from electron-hole exchange is different between SAQDs with $\mathrm{C}_{2 \mathrm{v}}$ symmetry and pyramidal QDs with a higher symmetry $\left(\mathrm{C}_{3 \mathrm{v}}\right)$ : In the former case, there is a splitting of the doubly degenerate bright states arising from the atomistic symmetry while, in the later case, this splitting does not exist. This symmetry analysis is not modified in the presence of strain induced by the lattice mismatch between InGaAs and GaAs because strain reduces the $T_{d}$ point group symmetry of the bulk materials to $\mathrm{C}_{3 \mathrm{v}}$ for heterostructures grown along the [111] axis. $^{45}$ The observation of a splitting between the bright exciton states is then entirely caused by a distortion of the pyramidal shape for dots with $\mathrm{C}_{3 \mathrm{v}}$ symmetry.

The comparison of the electron effective $g$ factors measured for $\mathrm{X}$ and $\mathrm{X}^{-}$in the same QD reveals a significant difference, which is much larger than the estimated experimental uncertainty. The effective $g$ factor is in a simplified picture a weighted average between the $g$ factors in the barrier and dot materials. ${ }^{46}$ It is then affected by a tiny modification of the wave function penetration into the barrier, which can take place due to the Coulomb repulsion between the two electrons. As a result, one would expect the electron effective $g$ factor in the dot to tend to its value in the barrier material. Because $g_{e}$ is positive in the barrier made of $\mathrm{Al}_{0.7} \mathrm{Ga}_{0.3} \mathrm{As},{ }^{47}$ the observed decrease in the modulus of $g_{e}$, when measured for $\mathrm{X}^{-}$, is consistent with our initial choice of a negative sign for the electron $g$ factor. We also emphasize that the relative change in the hole effective $g$ factor measured for $\mathrm{X}$ and $\mathrm{X}^{-}$is significant enough. A detailed explanation of this change would, however, go beyond a simple distortion of the hole wave function as the one described for the electron. We suggest instead that a mixing between the ground state and the excited states of the hole is taking place due to Coulomb correlations, which could lead to a contribution from higher orbital momenta to the groundstate envelope wave function and, then, to an increase in the hole effective $g$ factor. ${ }^{48}$

A discussion of our results with respect to experimental findings of the Zeeman splittings in strained SAQDs is appropriate. Both the Faraday and Voigt configurations were employed to investigate the optical anisotropy and the effective carrier $g$ factors of QDs in both II-VI and III-V material systems (Refs. 11, 14, and 15). These studies reported a strong optical anisotropy, that is, manifested by a high degree of linear polarization for both neutral and charged excitons. In contrast with these findings we measured a zero degree of linear polarization in the emission of our pyramidal QDs from the ground state. ${ }^{49}$ This behavior stems from the isotropy of the in-plane electric dipole moment since the two components belong to the same $\Gamma_{3}$ representation of the $C_{3 \mathrm{v}}$ point group symmetry. The optical anisotropy of the dipole moment in strained SAQDs results from the lower symmetry of the $\mathrm{C}_{2 \mathrm{v}}$ point group, for which the in-plane components of the dipole moment belong to different representations $\left(\Gamma_{3}\right.$ and $\Gamma_{4}$ ). The resulting ellipticity of the in-plane polarization was attributed to valence-band mixing induced by strain and confinement. In the Voigt configuration, the optical transition is split into a quadruplet of lines at a finite magnetic field, which is a behavior common to excitons and charged excitons confined to a quantum well ${ }^{50,51}$ or to a QD (Refs. 14, 15, and 17). An important difference exists, however, between the results in QDs and ours: we observe that the linear polarizations are oriented either parallel or perpendicular to the magnetic field when $B$ is parallel to [1-10] (Ref. 52) while there is no correlation with the field orientation with strained SAQDs. This is a consequence of the isotropy (anisotropy) of the in-plane $g$ factors in the $\mathrm{C}_{3 \mathrm{v}}\left(\mathrm{C}_{2 \mathrm{v}}\right)$ point group symmetry. Carrier effective $g$ factors have been obtained in the Voigt configuration from an analysis of the Zeeman splittings of the neutral exciton transitions (Refs. 11, 14, and 17); however, one of the dark-related exciton transitions cannot always be observed ${ }^{53}$ when $\left|g_{x}^{e}\right| \approx\left|g_{x}^{h}\right|$, which makes a separate determination of the carrier $g$ factors unprecise. It is, then, advantageous to evaluate the $g$ factors from the Zeeman splittings of the charged exciton transitions, which have similar emission intensities. This was realized in QDs from the emission of the negatively (Ref. 14) or positively (Ref. 15) charged exciton and in a single CdTe/ZnTe QD from the emission of negatively and positively charged excitons (Ref. 17). A separate determination of the electron and hole $g$ factors was carried out on the premises that the electron $g$ factor was isotropic (i.e., independent from the magnetic field orientation) and had the largest value. The general validity of these premises should be questioned, however, since the theoretical calculations of Pryor et al. ${ }^{34}$ predicted a large anisotropy of the electron $g$ factor in strained InAs/GaAs QDs. Furthermore, the in-plane hole $g$ factor could be larger depending on the size and shape asymmetry of the QDs. Nevertheless, using these premises the authors of Ref. 17 found a significant variation in the hole in-plane $g$ factor with the sign of the charged exciton and attributed this effect to an enhancement of the hole confinement caused by the Coulomb attraction with electrons in the QD. This mechanism should be distinguished from the Coulomb correlations between the few carriers composing the charged excitonic species. Modifications of the exciton wave function upon charging a QD with a hole has also been studied in $\operatorname{In}(\mathrm{Ga})$ As QDs by probing the permanent excitonic dipole moment of neutral and charged excitons. ${ }^{54}$ The observation of a different dipole moment was attributed to a lateral redistribution of the hole wave function in the plane of the dot upon charging the QD with an additional hole on the basis of a few-body calculation of the excitonic states. This conclusion is surprising in view of the discrepancy between the calculated and measured values of the reduction in the excitonic dipole moment upon charging the QD with one hole or with two holes.

We believe that our results will spur theoretical works to investigate the interplay between Coulomb correlations ${ }^{55-57}$ and the Zeeman interaction in semiconductor QDs in order to quantify effects of Coulomb correlations in charged excitonic complexes. Further investigations on the theoretical side could examine the anisotropy (in plane versus out of plane) of the carrier $g$ factors and their signs for QDs with varying parameters describing the shape, composition profile, and size. We add that investigations of the $g$ factors in $\operatorname{In}(\mathrm{Ga}) \mathrm{As}$ SAQDs of $\mathrm{C}_{2 \mathrm{v}}$ symmetry have already provided useful insight to understand the effect of strain on the $g$ factors mea- 
sured in the Faraday configuration. ${ }^{58}$ On the experimental side, the pyramidal QDs with $\mathrm{C}_{3 \mathrm{v}}$ symmetry are promising candidates for realizing a source of entangled photon pairs ${ }^{59}$ since the degeneracy of the bright exciton states is imposed by the $\mathrm{C}_{3 \mathrm{v}}$ symmetry while it is lifted in pyramidal QDs with $\mathrm{C}_{2 \mathrm{v}}$ symmetry. Entanglement of polarized photon pairs have been demonstrated in specific cases, however, by using a spectral filtering scheme ${ }^{60}$ or by selecting QDs with quasizero fine structure spin splitting or, alternatively, by applying an in-plane magnetic field. ${ }^{61}$ The entanglement of photon pairs in QDs with $\mathrm{C}_{3 \mathrm{v}}$ symmetry was also predicted independently by Singh and Bester. ${ }^{62}$ Pyramidal QDs with $\mathrm{C}_{3 \mathrm{v}}$ symmetry could be also used to initialize an electron-spin state simultaneously on several QDs by optical pumping of the charged exciton transitions in a transverse magnetic field. This would solve the issue of scalability encountered in the recent demonstrations of a single spin control and initialization in QDs (Refs. 5 and 8) and open the way to quantum information processing at the level of several qubits (Ref. 3).

In summary, we presented a simple approach to investigate the carrier effective $g$ factors in pyramidal QDs that relied on the Zeeman energy splittings of the charged excitons in a Voigt configuration. We studied the optical emission of pyramidal In $(\mathrm{Ga}) \mathrm{As}$ QDs with $\mathrm{C}_{3 \mathrm{v}}$ symmetry. We observed that the effective $g$ factors measured in this way depended on the sign of the charged exciton, revealing the role of Coulomb correlations between the carriers confined to the QD. We discussed the magneto-optical properties of pyramidal QDs in relation to their symmetry and showed that strain did not introduce any optical anisotropy in the plane of the pyramid for QDs with $\mathrm{C}_{3 \mathrm{v}}$ symmetry.

\section{ACKNOWLEDGMENT}

We are grateful to M.-A. Dupertuis for stimulating discussions on symmetries.
*Present address: Tyndall National Institute, Lee Maltings, Prospect Row, Cork, Ireland.

${ }^{1}$ D. Loss and D. P. DiVincenzo, Phys. Rev. A 57, 120 (1998).

${ }^{2}$ A. Imamoglu, D. D. Awschalom, G. Burkard, D. P. DiVincenzo, D. Loss, M. Sherwin, and A. Small, Phys. Rev. Lett. 83, 4204 (1999).

${ }^{3}$ S. M. Clark, Kai-Mei C. Fu, T. D. Ladd, and Y. Yamamoto, Phys. Rev. Lett. 99, 040501 (2007).

${ }^{4}$ M. Atatüre, J. Dreiser, A. Badolato, A. Högele, K. Karrai, and A. Imamoglu, Science 312, 551 (2006).

${ }^{5}$ X. Xu, Y. Wu, Bo Sun, Q. Huang, J. Cheng, D. G. Steel, A. S. Bracker, D. Gammon, C. Emary, and L. J. Sham, Phys. Rev. Lett. 99, 097401 (2007).

${ }^{6}$ B. D. Gerardot, D. Brunner, P. A. Dalgarno, P. Öhberg, S. Seidl, M. Kroner, K. Karrai, N. G. Stoltz, P. M. Petroff, and R. J. Warburton, Nature (London) 451, 441 (2008).

${ }^{7}$ A. J. Ramsay, S. J. Boyle, R. S. Kolodka, J. B. B. Oliveira, J. Skiba-Szymanska, H. Y. Liu, M. Hopkinson, A. M. Fox, and M. S. Skolnick, Phys. Rev. Lett. 100, 197401 (2008).

${ }^{8}$ D. Press, T. D. Ladd, B. Zhang, and Y. Yamamoto, Nature (London) 456, 218 (2008).

${ }^{9}$ Pochung Chen, C. Piermarocchi, L. J. Sham, D. Gammon, and D. G. Steel, Phys. Rev. B 69, 075320 (2004).

${ }^{10}$ G. Medeiros-Ribeiro, M. V. B. Pinheiro, V. L. Pimentel, and E. Marega, Appl. Phys. Lett. 80, 4229 (2002).

${ }^{11}$ M. Bayer, G. Ortner, O. Stern, A. Kuther, A. A. Gorbunov, A. Forchel, P. Hawrylak, S. Fafard, K. Hinzer, T. L. Reinecke, S. N. Walck, J. P. Reithmaier, F. Klopf, and F. Schäfer, Phys. Rev. B 65, 195315 (2002), and references therein.

${ }^{12}$ T. Nakaoka, T. Saito, J. Tatebayashi, and Y. Arakawa, Phys. Rev. B 70, 235337 (2004).

${ }^{13}$ M. Sénès, B. Urbaszek, X. Marie, T. Amand, J. Tribollet, F. Bernardot, C. Testelin, M. Chamarro, and J. M. Gérard, Phys. Rev. B 71, 115334 (2005).

${ }^{14}$ A. V. Koudinov, I. A. Akimov, Y. G. Kusrayev, and F. Henneberger, Phys. Rev. B 70, 241305(R) (2004).

${ }^{15}$ D. N. Krizhanovskii, A. Ebbens, A. I. Tartakovskii, F. Pulizzi,
T. Wright, M. S. Skolnick, and M. Hopkinson, Phys. Rev. B 72, 161312(R) (2005).

${ }^{16}$ T. Kiessling, A. V. Platonov, G. V. Astakhov, T. Slobodskyy, S. Mahapatra, W. Ossau, G. Schmidt, K. Brunner, and L. W. Molenkamp, Phys. Rev. B 74, 041301(R) (2006).

${ }^{17}$ Y. Léger, L. Besombes, L. Maingault, and H. Mariette, Phys. Rev. B 76, 045331 (2007).

${ }^{18}$ B. Urbaszek, R. J. Warburton, K. Karrai, B. D. Gerardot, P. M. Petroff, and J. M. Garcia, Phys. Rev. Lett. 90, 247403 (2003).

${ }^{19}$ M. Ediger, G. Bester, A. Badolato, P. M. Petroff, K. Karrai, A. Zunger, and R. J. Warburton, Nat. Phys. 3, 774 (2007).

${ }^{20}$ A. Hartmann, Y. Ducommun, E. Kapon, U. Hohenester, and E. Molinari, Phys. Rev. Lett. 84, 5648 (2000).

${ }^{21}$ R. J. Warburton, C. Schäflein, D. Haft, F. Bickel, A. Lorke, K. Karrai, J. M. Garcia, W. Schoenfeld, and P. M. Petroff, Nature (London) 405, 926 (2000).

${ }^{22}$ D. V. Regelman, E. Dekel, D. Gershoni, E. Ehrenfreund, A. J. Williamson, J. Shumway, A. Zunger, W. V. Schoenfeld, and P. M. Petroff, Phys. Rev. B 64, 165301 (2001).

${ }^{23}$ S. Rodt, A. Schliwa, K. Pötschke, F. Guffarth, and D. Bimberg, Phys. Rev. B 71, 155325 (2005).

${ }^{24}$ M. H. Baier, A. Malko, E. Pelucchi, D. Y. Oberli, and E. Kapon, Phys. Rev. B 73, 205321 (2006).

${ }^{25}$ A. Malko, D. Y. Oberli, M. H. Baier, E. Pelucchi, F. Michelini, K. F. Karlsson, M.-A. Dupertuis, and E. Kapon, Phys. Rev. B 72, 195332 (2005).

${ }^{26}$ K. V. Kavokin, Phys. Status Solidi A 195, 592 (2003).

${ }^{27}$ E. L. Ivchenko and G. Pikus, Superlattices and Other Heterostructures (Springer-Verlag, Berlin, 1995), Vol. 110.

${ }^{28}$ For an application of the method of invariants, see also: A. K. Bhattacharjee and S. Rodriguez, Phys. Rev. B 6, 3836 (1972).

${ }^{29}$ K. Cho, S. Suga, W. Dreybrodt, and F. Willmann, Phys. Rev. B 11, 1512 (1975).

${ }^{30}$ Using the method of invariants, the representation of the Zeeman Hamiltonian in the spinor basis states of the irreducible representation, $\Gamma_{4}$, is a $2 \times 2$ matrix, which is expressed as a linear combination of the Pauli spin matrices with equal coefficients in 
front of $\sigma_{x}$ and $\sigma_{y}$ since $\sigma_{x}$ and $\sigma_{y}$ transform according to the same irreducible representation $\Gamma_{3}$ and a different coefficient in front of $\sigma_{z}$ since $\sigma_{z}$ transforms according to another irreducible representation $\Gamma_{2}$ of $\mathrm{C}_{3 \mathrm{v}}$.

${ }^{31}$ If the hole state belong to either one or other one-dimensional representation of $\mathrm{C}_{3 \mathrm{v}}, \Gamma_{5}$, or $\Gamma_{6}$, a magnetic field applied in the $(x, y)$ plane does not lift the degeneracy of the Kramers degenerate hole states $\left(g_{x}^{h}=g_{y}^{h}=0\right)$. A magnetic field applied along the $z$ axis lifts this degeneracy and introduces a linear dependence with $B_{z}\left(g_{z}^{h} \neq 0\right)$ in the Zeeman Hamiltonian.

${ }^{32}$ D. Gammon, E. S. Snow, B. V. Shanabrook, D. S. Katzer, and D. Park, Phys. Rev. Lett. 76, 3005 (1996).

${ }^{33}$ E. Poem, J. Shemesh, I. Marderfeld, D. Galushko, N. Akopian, D. Gershoni, B. D. Gerardot, A. Badolato, and P. M. Petroff, Phys. Rev. B 76, 235304 (2007).

${ }^{34}$ C. E. Pryor and M. E. Flatté, Phys. Rev. Lett. 96, 026804 (2006); 99, 179901(E) (2007).

${ }^{35}$ W. Sheng, S. J. Xu, P. Hawrylak, Phys. Rev. B 77, 241307(R) (2008).

${ }^{36}$ M. Byszewski, B. Chalupar, K. F. Karlsson, D. Y. Oberli, E. Pelucchi, A. Rudra, and E. Kapon, Physica E (Amsterdam) 40, 1873 (2008).

${ }^{37}$ P.-F. Braun, B. Urbaszek, T. Amand, X. Marie, O. Krebs, B. Eble, A. Lemaitre, and P. Voisin, Phys. Rev. B 74, 245306 (2006); P. Maletinsky, C. W. Lai, A. Badolato, and A. Imamoglu, ibid. 75, 035409 (2007).

${ }^{38}$ J. J. Finley, D. J. Mowbray, M. S. Skolnick, A. D. Ashmore, C. Baker, A. F. G. Monte, and M. Hopkinson, Phys. Rev. B 66, 153316 (2002).

${ }^{39}$ C. Schulhauser, A. Högele, R. J. Warburton, A. O. Govorov, W. Schoenfeld, J. M. Garcia, P. M. Petroff, and K. Karrai, Phys. Status Solidi B 238, 293 (2003).

${ }^{40}$ The spinor basis for the degenerate hole states belonging to $\Gamma_{5}$ and $\Gamma_{6}$ has the explicit form given by $\left\{\frac{1}{\sqrt{2}}\left(\left|\frac{3}{2}, \frac{3}{2}\right\rangle\right.\right.$ $\left.\left.-\left|\frac{3}{2},-\frac{3}{2}\right\rangle\right), \frac{1}{\sqrt{2}}\left(\left|\frac{3}{2}, \frac{3}{2}\right\rangle+\left|\frac{3}{2},-\frac{3}{2}\right\rangle\right)\right\}$.

${ }^{41}$ If the hole state belong to either one or other one-dimensional representation of $\mathrm{C}_{3 \mathrm{v}}, \Gamma_{5}$, or $\Gamma_{6}$, the neutral exciton states belong to $\Gamma_{3}$ (since $\Gamma_{4} \otimes \Gamma_{5}=\Gamma_{3}$ and $\Gamma_{4} \otimes \Gamma_{6}=\Gamma_{3}$ ) and, thus, are split into two doubly degenerate states, which means that $\delta=\delta^{*}=0$ in the expression of the exchange Hamiltonian.
${ }^{42}$ R. Romestain and G. Fishman, Phys. Rev. B 49, 1774 (1994).

${ }^{43}$ E. L. Ivchenko, Phys. Status Solidi A 164, 487 (1997).

${ }^{44}$ G. Bester, S. Nair, and A. Zunger, Phys. Rev. B 67, 161306(R) (2003).

${ }^{45}$ C. Mailhiot and D. L. Smith, Phys. Rev. B 35, 1242 (1987).

${ }^{46}$ This picture is expected to be valid in a perturbative limit and cannot be employed to evaluate the $g$ factors in heterostructures from the effective $g$ factors of the bulk materials.

${ }^{47}$ P. Pfeffer and W. Zawadzki, Phys. Rev. B 74, 115309 (2006).

${ }^{48}$ W. Sheng and A. Babinski, Phys. Rev. B 75, 033316 (2007).

${ }^{49}$ K. F. Karlsson, V. Troncale, D. Y. Oberli, A. Malko, E. Pelucchi, A. Rudra, and E. Kapon, Appl. Phys. Lett. 89, 251113 (2006).

${ }^{50}$ J. G. Tischler, A. S. Bracker, D. Gammon, and D. Park, Phys. Rev. B 66, 081310(R) (2002).

${ }^{51}$ I. Toft and R. T. Phillips, Phys. Rev. B 76, 033301 (2007).

${ }^{52}$ In the general case, one can easily show that the linear polarization axes $(x$ and $y)$ are rotated by an angle $\theta$ for an arbitrary rotation of the magnetic field around the $z$ axis by minus $\theta$ starting from the $x$ axis.

${ }^{53}$ R. M. Stevenson, R. J. Young, P. See, D. G. Gevaux, K. Cooper, P. Atkinson, I. Farrer, D. A. Ritchie, and A. J. Shields, Phys. Rev. B 73, 033306 (2006).

${ }^{54}$ J. J. Finley, M. Sabathil, P. Vogl, G. Abstreiter, R. Oulton, A. I. Tartakovskii, D. J. Mowbray, M. S. Skolnick, S. L. Liew, A. G. Cullis, and M. Hopkinson, Phys. Rev. B 70, 201308(R) (2004).

${ }^{55}$ A. Barenco and M. A. Dupertuis, Phys. Rev. B 52, 2766 (1995).

${ }^{56}$ J. Shumway, A. Franceschetti, and Alex Zunger, Phys. Rev. B 63, 155316 (2001).

${ }^{57}$ G. Bester and A. Zunger, Phys. Rev. B 68, 073309 (2003).

${ }^{58}$ T. Nakaoka, T. Saito, J. Tatebayashi, S. Hirose, T. Usuki, N. Yokoyama, and Y. Arakawa, Phys. Rev. B 71, 205301 (2005).

${ }^{59}$ O. Benson, C. Santori, M. Pelton, and Y. Yamamoto, Phys. Rev. Lett. 84, 2513 (2000).

${ }^{60}$ N. Akopian, N. H. Lindner, E. Poem, Y. Berlatzky, J. Avron, D. Gershoni, B. D. Gerardot, and P. M. Petroff, Phys. Rev. Lett. 96, 130501 (2006).

${ }^{61}$ R. M. Stevenson, R. J. Young, P. Atkinson, K. Cooper, D. A. Ritchie, and A. J. Shields, Nature (London) 439, 179 (2006).

${ }^{62}$ R. Singh and G. Bester, Phys. Rev. Lett. 103, 063601 (2009). 